\title{
Data protection, ePrivacy, and the prospects for Apple's on-device CSAM Detection system in Europe
}

Jennifer Cobbe*

\begin{abstract}
In this paper, I assess what EU data protection and ePrivacy law might say about Apple's on-device CSAM Detection system. I provide a high-level overview of the CSAM Detection system (hopefully accessible to non-tech people), an analysis of how it relates to EU data protection law (hopefully accessible to non-legal people), and some further discussion based on this analysis. I conclude that EU law currently presents obstacles that might slow or preclude the deployment of this on-device CSAM Detection system in the EU. However, these barriers may be removed by future EU or Member State legislation.
\end{abstract}

\section{Introduction}

Apple recently confirmed ${ }^{1}$ that it plans to include on-device detection of child sexual abuse material (CSAM) in its next iOS and iPadOS updates, due to roll out later this year. Apple's CSAM Detection system would use on-device processing to analyse images to be uploaded by iPhone users of its iCloud Photos backup service ${ }^{2}$. In this paper, I attempt to assess what EU data protection and ePrivacy law might say about this CSAM Detection system. I conclude that EU law currently presents obstacles that might slow or preclude the deployment of this on-device CSAM Detection system in the EU.

In my view, set out in this analysis, the principal obstacle is that, as it stands, EU law would require that Apple obtain the consent of individual iPhone users for on-device scanning. This consent would need to be opt-in, rather than opt-out; there would need to be a real possibility for users to refuse consent; users' access to iCloud could not be made conditional

\footnotetext{
* Compliant and Accountable Systems Group, Department of Computer Science and Technology, University of Cambridge (jennifer.cobbe@cst.cam.ac.uk). Thanks to Jat Singh and Jovan Power for patient advice on how the CSAM Detection system works.

${ }^{1}$ Apple, 'Expanded protections for children' (2021) < https://www.apple.com/child-safety> accessed August 8th 2021.

${ }^{2}$ Apple, 'CSAM Detection: Technical Summary' (2021) < https://www.apple.com/childsafety/pdf/CSAM Detection Technical Summary.pdf> accessed August 8th 2021.
} 
on giving consent to CSAM Detection; and users must be able to withdraw consent without suffering loss of iCloud service. This may place a welcome brake on the deployment of ondevice CSAM detection in the EU. However, these barriers may be removed by future EU or Member State legislation ${ }^{3}$ - just as similar potential barriers for automated CSAM detection by certain messaging services have already been removed by EU legislation ${ }^{4}$.

It's important to note here that Apple says that this CSAM Detection system will only be deployed in the US ${ }^{5}$. So, for now, the EU law analysis presented here is speculative, assessing what the situation would be if Apple decided to deploy it in the EU as well. My understanding of how Apple's CSAM Detection system works is based on the Technical Summary made available online by Apple ${ }^{6}$.

Here, I try to provide a high-level overview of Apple's CSAM Detection system (hopefully accessible to non-tech people), an analysis of how it relates to EU data protection law (hopefully accessible to non-legal people), and some further discussion based on this analysis. I'Il add here that I'm setting out my understanding of how Apple's system is intended to work, of how the law might apply to it, and of what the implications of that might be. This has not been peer reviewed and shouldn't be taken as a definitively correct analysis - if you think I've got something wrong, then please get in touch. Any mistakes or errors here are mine alone; based on feedback, I may amend or revise this paper if needed.

(Note that this analysis applies equally to the UK, which carried both the General Data Protection Regulation ${ }^{7}$ and the regulations ${ }^{8}$ implementing the related ePrivacy Directive ${ }^{9}$ over into its post-Brexit domestic law through the European Union (Withdrawal) Act 2018. Two regulations were made - one in $2019^{10}$ and another in $2020^{11}$ - to amend both GDPR and the regulations implementing the ePrivacy Directive so as to account for Brexit. Where I

\footnotetext{
${ }^{3}$ Directive 2002/58/EC of the European Parliament and of the Council of 12 July 2002 concerning the processing of personal data and the protection of privacy in the electronic communications sector (Directive on privacy and electronic communications) [2002] OJ L 201/37 ('ePrivacy Directive') art 15(1).

${ }^{4}$ Regulation (E) 2021/1232 of the European Parliament and of the Council of 14 July 2021 on a temporary derogation from certain provisions of Directive 2002/58/EC as regards the use of technologies by providers of number-independent interpersonal communications services for the processing of personal and other data for the purpose of combating online child sexual abuse [2020] OJ L 274/41.

${ }^{5}$ Chance Miller, 'Apple says any expansion of CSAM detection outside of the US will occur on a per-country basis' (2021) 9to5Mac <https://9to5mac.com/2021/08/06/apple-says-any-expansion-of-csam-detectionoutside-of-the-us-will-occur-on-a-per-country-basis> accessed August 9th 2021.

${ }^{6}$ Apple, Technical Summary (n 2).

${ }^{7}$ Regulation (EU) 2016/679 of the European Parliament and of the Council of 27 April 2016 on the protection of natural persons with regard to the processing of personal data and on the free movement of such data, and repealing Directive 95/46/EC (General Data Protection Regulation) [2016] OJ L119/1 ('GDPR').

8 The Privacy and Electronic Communications (EC Directive) Regulations 2003.

${ }^{9}$ ePrivacy Directive.

${ }^{10}$ The Data Protection, Privacy and Electronic Communications (Amendments etc) (EU Exit) Regulations 2019.

${ }^{11}$ The Data Protection, Privacy and Electronic Communications (Amendments etc)(EU Exit) Regulations 2020.
} 
say 'EU', you can generally read 'UK' without going too far wrong, but be aware that there may be some differences in detail ${ }^{12}$ ).

\section{How does Apple's CSAM Detection system work?}

Apple's Technical Summary says:

"CSAM Detection enables Apple to accurately identify and report iCloud users who store known Child Sexual Abuse Material (CSAM) in their iCloud Photos accounts. Apple servers flag accounts exceeding a threshold number of images that match a known database of CSAM image hashes so that Apple can provide relevant information to the National Center for Missing and Exploited Children (NCMEC). This process is secure, and is expressly designed to preserve user privacy"13.

At a high level, Apple believes that it can identify CSAM uploaded by iCloud users by comparing users' uploaded images with a database of known CSAM. Apple claims that it can do this by automatically converting images into 'hashes' - numeric strings representing each image individually. To do this, it uses a technology it calls 'NeuralHash'14. In Apple's words, the NeuralHash function "maps images to numbers" 15 . If two separate files containing the same image are hashed, the numeric strings produced for each file should be the same ${ }^{16}$. That is to say, by comparing the hashes produced for two separate files, Apple says, it is possible to determine whether they contain the same image ${ }^{17}$.

I'll now attempt a more detailed description of how Apple tries to do this. Using the NeuralHash function, Apple first converts the known CSAM in the NCMEC database into a set of NeuralHashes, each representing an individual known CSAM image ${ }^{18}$. These NeuralHashes are then 'blinded' by applying a secret blinding key known only to Apple ${ }^{19}$. These blinded hashes are stored in a hash table ${ }^{20}$ (i.e. a table containing rows of blinded hashes). Row numbers in that hash table are derived from unblinded NeuralHashes ${ }^{21}$ (see

\footnotetext{
${ }^{12}$ A consolidated version of the UK GDPR - incorporating amendments made by the 2019 and 2020 regulations - is available for reference here: https://www.mishcon.com/uk-gdpr.

${ }^{13}$ Apple, Technical Summary (n 2), p.3.

${ }^{14}$ Apple, Technical Summary (n 2), pp.5-6.

${ }^{15}$ Apple, Technical Summary (n 2), p.5.

${ }^{16}$ Apple, Technical Summary (n 2), p.5.

${ }^{17}$ Apple, Technical Summary (n 2), p.5.

${ }^{18}$ Apple, Technical Summary (n 2), p.6.

${ }^{19}$ Apple, Technical Summary (n 2), p.6.

${ }^{20}$ Apple, Technical Summary (n 2), p.6.

${ }^{21}$ Apple, Technical Summary (n 2), p.6.
} 
Figure 1). This hash table is then stored on users' iPhones $^{22}$ (Apple says it will include CSAM Detection in iPadOS, so where I say 'iPhone' in this paper I could equally say 'iPad').

When a user attempts to upload an image to iCloud (either through automatic or manual backup), Apple converts the image into a NeuralHash using on-device processing (i.e. processing on the user's iPhone $)^{23}$. The iPhone then checks the image's NeuralHash against entries in the hash table so that it can create an encryption key ${ }^{24}$. If the NeuralHash matches an entry in the table, the corresponding blinded hash can be returned. Importantly, though blinded hashes can only be produced by one NeuralHash (using Apple's secret blinding key), the hash table may return the same blinded hash in response to many different NeuralHashes, including NeuralHashes corresponding to images that do not contain CSAM.

The device then derives an encryption key from the returned blinded hash ${ }^{25}$. The device uses this key to encrypt a 'payload' containing the NeuralHash and a representation of the scanned image ${ }^{26}$. This payload can only be decrypted with a decryption key derived from the original NeuralHash that was combined with Apple's secret blinding key to produce the blinded hash from which the encryption key was itself derived.

Separately, using a different function, the device uses the NeuralHash to produce a cryptographic 'header' (essentially, a string corresponding to the NeuralHash). This header corresponds only to the NeuralHash for the user's image. The header and the encrypted payload are then combined into a 'safety voucher', which is uploaded to Apple's server along with the underlying image ${ }^{27}$

Using the safety voucher's cryptographic header, Apple's server then produces a decryption $k^{2} y^{28}$. Because the header corresponds only to the NeuralHash used to create it (which itself corresponds only to the underlying hashed image), this decryption key will be unique to each image. For the decryption key to work, the NeuralHash from which it was ultimately derived must be the same as the NeuralHash from which the encryption key was ultimately derived. That is to say, to decrypt the payload, NeuralHash of the user's image must match the NeuralHash of a known CSAM image ${ }^{29}$. If those NeuralHashes do not match, then the payload cannot be decrypted ${ }^{30}$.

\footnotetext{
${ }^{22}$ Apple, Technical Summary (n 2), p.6.

${ }^{23}$ Apple, Technical Summary (n 2), p.7.

${ }^{24}$ Apple, Technical Summary (n 2), p.7.

${ }^{25}$ Apple, Technical Summary (n 2), p.7.

${ }^{26}$ Apple, Technical Summary (n 2), p.4, p.7, pp.9-11.

${ }^{27}$ Apple, Technical Summary (n 2), pp.7-8.

${ }^{28}$ Apple, Technical Summary (n 2), p.7.

${ }^{29}$ Apple, Technical Summary (n 2), p.7.

${ }^{30}$ Apple, Technical Summary (n 2), p.7.
} 


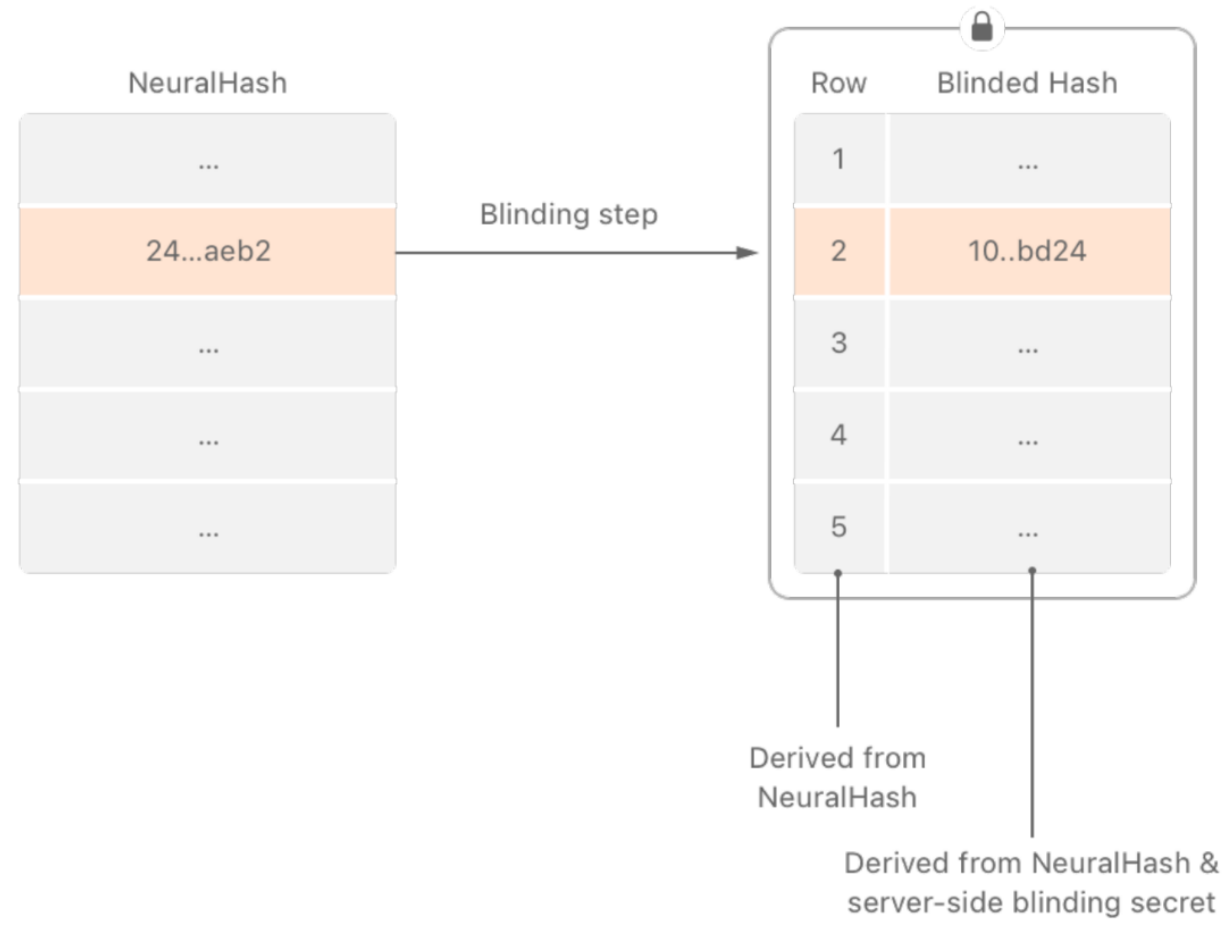

Figure 1. Apple's depiction of how hashes and blinded hashes are together used to create the database that is stored on users' devices (taken from Apple, Technical Summary, p.6). Row numbers are derived from unblinded hashes. Blinded hashes are stored alongside them.

This process is an example of a cryptographic protocol called 'Private Set Intersection', used here to determine whether upload images correspond to known CSAM $^{31}$. Using this, Apple can - in theory - determine whether any of the images to be uploaded match any of the images in the NCMEC database ${ }^{32}$. Apple says that its hash matching works irrespective of whether images are cropped or are different sizes or resolutions ${ }^{33}$.

Another technology, 'Threshold Secret Sharing', ensures that Apple can't actually look inside safety vouchers unless the associated iCloud account uploads a pre-determined number of positive matches ${ }^{34}$. If that threshold is crossed, Apple can inspect the contents of the vouchers associated with the account ${ }^{35}$. This involves manual review of vouchers and their contents, including the representations of underlying images ${ }^{36}$. If CSAM is found, the account is disabled, and a report is made to the NCMEC ${ }^{37}$. Through this CSAM Detection

\footnotetext{
${ }^{31}$ Apple, Technical Summary (n 2), pp.6-10; see also Apple, 'Apple PSI System: Security Protocol and Analysis' (2021) <https://www.apple.com/child-safety/pdf/Apple PSI_System Security Protocol and Analysis.pdf> accessed August 8th 2021.

${ }^{32}$ Apple, Technical Summary (n 2), pp.5-6.

${ }^{33}$ Apple, Technical Summary (n 2), p.5.

${ }^{34}$ Apple, Technical Summary (n 2), pp.7-12.

${ }^{35}$ Apple, Technical Summary (n 2), p.8.

${ }^{36}$ Apple, Technical Summary (n 2), p.4.

${ }^{37}$ Apple, Technical Summary (n 2), p.4.
} 
method, Apple says that it can cryptographically ensure that it only learns anything about user's images that match those in the database, and nothing about images that don' $t^{38}$.

The process is, of course, more complicated than this brief overview suggests. But we only need to know the outline of what is happening here, rather than the precise technical details. Following the above, there are broadly three components to what Apple intends to do. First, the on-device (i.e. on-iPhone) aspects of the matching process that hashes images and uploads safety vouchers to the server (involving NeuralHash and Private Set Intersection). Second, the on-server determinations of whether the safety voucher contains a positive match, and of whether the account has crossed the threshold for manual review (involving Private Set Intersection and Threshold Secret Sharing). Third, the decision of whether to refer a particular account to the NCMEC (involving manual review of safety vouchers).

\section{Would GDPR apply to this?}

Broadly, with some exceptions, GDPR applies to the processing of personal data ${ }^{39}$. GDPR sets out both a 'material scope' 40 (what kinds of activity it applies to) and a 'territorial scope $^{41}$ (where those activities should take place geographically for GDPR to apply). For GDPR to apply, the activity in question must be within both its material scope and its territorial scope. Let's look at each of these in more detail.

\subsection{Material scope}

GDPR says that it applies to the processing of personal data wholly or partly by automated means ${ }^{42}$. Most relevant to us is that the on-device and on-server components of Apple's CSAM Detection system are wholly automated. The only human involvement comes in the third component, at the end of the process, where a user's account that has met the threshold is manually reviewed. As that manual review would involve looking at files on a computer, it is partly automated. The CSAM Detection system in its entirety thus involves 'wholly or partly automated means'.

\footnotetext{
${ }^{38}$ Apple, Technical Summary (n 2), p.12.

${ }^{39}$ GDPR art 1.

40 GDPR art 2

${ }^{41}$ GDPR art 3.

${ }^{42}$ GDPR art 3; GDPR also applies to non-automated means where personal data forms or is intended to form part of a filing system.
} 
Processing is defined in GDPR to mean:

"any operation or set of operations which is performed on personal data or on sets of personal data, whether or not by automated means, such as collection, recording, organisation, structuring, storage, adaptation or alteration, retrieval, consultation, use, disclosure by transmission, dissemination or otherwise making available, alignment or combination, restriction, erasure or destruction" 43

This is a long and wordy definition, but I've bolded the most important part. Essentially, in law, 'processing' means anything you do with personal data. Collecting personal data is processing. Analysing personal data is processing. Encrypting personal data is processing. Sharing personal data is processing. Moving personal data from device to server is processing. Deleting personal data is processing. Even just storing personal data on a drive and leaving it alone is processing.

As described above, based on Apple's documentation, Apple's CSAM Detection system converts images into hashes. It analyses those hashes by comparing them with known CSAM. A representation of each image and the NeuralHash of the image are then combined into a safety voucher, which cryptographically encodes the outcome of the hash matching process. This safety voucher is uploaded to Apple's servers. Where a threshold number of positive matches is reached, those safety vouchers are decrypted and inspected. Apple's CSAM Detection system thus involves many different operations performed on data, constituting various kinds of processing.

The next question is whether that data is personal data. GDPR defines personal data as:

\footnotetext{
"any information relating to an identified or identifiable natural person ('data subject'); an identifiable natural person is one who can be identified, directly or indirectly, in particular by reference to an identifier such as a name, an identification number, location data, an online identifier or to one or more factors specific to the physical, physiological, genetic, mental, economic, cultural or social identity of that natural person" 44
}

Again, this is a long and wordy definition, and again I have bolded the most important part. In essence, personal data is any information that can in some way be linked with a specific living individual ('natural person' is the law's term for an individual human ${ }^{45}$ ). The individual

\footnotetext{
${ }^{43}$ GDPR art 4(2).

${ }^{44}$ GDPR art 4(1); see also GDPR recitals 26, 30

45 This contrasts with a 'legal person', such as a corporation, which is artificially assigned some degree of legal personality by the law, allowing them to enter into contracts, own property, sue and be sued, and so on.
} 
to whom that information relates is known as the 'data subject'. An individual's personal details - such as their name or address - are obviously personal data. Other information as diverse as bank about details, tax records, or online account identifiers will also generally be personal data. Even an IP or MAC address may sometimes be personal data ${ }^{46}$. It does not matter whether the actual identity of the individual is known (i.e. their name, address, and so on), only that they can be distinguished from others.

Importantly, it is not necessary that the information itself directly identifies an individual ${ }^{47}$. The information may be linked to other information which allows an individual to be identified (a list of songs listened to by a user of a music streaming service, for example, will be personal data, as the list is linked to the account from which the user can be identified). Nor must all the information needed to identify an individual be held by one person ${ }^{48}$. That is to say, if information held by me could identify an individual when combined with other information held by someone else, then that information held by me can be personal data, even if that information does not by itself allow the individual to be identified.

It may also be the case that combining several pieces of information that are not otherwise personal data can result in personal data. An example here would be a data profile that allows an individual to be 'singled out' ${ }^{49}$. Individual data points in that profile like date of birth, gender, occupation, relationship status, and so on may not by themselves constitute personal data. But enough of those data points combined might allow you to pinpoint one individual and distinguish them from others. In that case, those data points, when taken together, will also be personal data.

The broad concept of personal data ${ }^{50}$ in EU law can be unintuitive for people familiar with the much narrower concept of 'Personally Identifiable Information' found in the United States. The important questions are, however, rather straightforward: (1) Does this information relate to a person? (2) Can that person be distinguished from others?

I'll now consider three things to determine whether they are personal data: users' images to be uploaded to iCloud; the NeuralHashes of those images; and the safety vouchers generated by the CSAM Detection system.

\footnotetext{
${ }^{46}$ GDPR recital 30.

${ }^{47}$ Case C-582/14 Breyerv Bundesrepublik Deutschland [2016] ('Breyer') para 41; GDPR recital 26.

${ }^{48}$ Breyer para 43.

${ }^{49}$ See Frederik J Zuiderveen Borgesius, 'Singling out people without knowing their names - Behavioural targeting, pseudonymous data, and the new Data Protection Regulation' [2016] Computer Law \& Security Review 32, 256-271; GDPR recital 26.

${ }^{50}$ Nadezhda Purtova, 'The law of everything. Broad concept of personal data and future of EU data protection law' (2018) 10 Law, Innovation and Technology <https://www.tandfonline.com/doi/full/10.1080/17579961.2018.1452176>.
} 
1. User images. Images are not necessarily themselves personal data. If they depict living people and it is clear who those people are then those images likely will be personal data (as they will then relate to people and those people will be identifiable). If the images do not depict living people then whether they are personal data or not will depend on whether they are in some other way linked to a living person. In the case of Apple's CSAM Detection system, images are processed prior to upload to iCloud and are associated with that particular iCloud account. All of the images scanned by CSAM Detection - whether containing CSAM or not would therefore be personal data relating to the iCloud account holder in question. They may also be personal relating to any people depicted in them.

2. NeuralHashes. Since the user's images are personal data, we need to consider whether hashes of those images are also personal data (relating either to the people depicted in the images or to the iCloud user). GDPR says that, in determining whether someone can be identified from information (such as by reversing a hash and viewing an image depicting them), we should take into account all means reasonably likely to lead to identification ${ }^{51}$. According to GDPR, in assessing whether there are means that are reasonably likely to lead to identification, we should consider all objective factors, including the cost of and time needed for identification, and should take into consideration both the available technology now and technological developments ${ }^{52}$. Apple's NeuralHash is a one-way function; that is to say, it is designed to be irreversible without prohibitive time and computing power. Though it may in some sense be theoretically possible to reverse a hash generated by Apple's NeuralHash process (given infinite time and computing power), this is not reasonably likely.

However, hashes are associated with a particular iCloud account. The images that the hashes represent are also associated with that same iCloud account. The hashing function (NeuralHash) is known to Apple. Images stored on iCloud Photos are encrypted for security reasons, but not end-to-end encrypted such that they are beyond the reach of Apple ${ }^{53}$ (which holds the decryption key offers access to US law enforcement ${ }^{54}$ ). It may therefore be possible for Apple to access and hash images stored on iCloud Photos and identify matching hashes (thereby linking those hashes with images that may depict individuals). As the images may be personal data of

\footnotetext{
${ }^{51}$ GDPR recital 26.

52 GDPR recital 26.

${ }^{53}$ Apple, 'iCloud Security Overview' <https://support.apple.com/en-us/HT202303> accessed August 10th 2021.

${ }^{54}$ Apple, 'Legal Process Guidelines: Government \& Law Enforcement within the United States', p. $<$ https://www.apple.com/legal/privacy/law-enforcement-guidelines-us.pdf> accessed August 10th 2021; see also Joseph Menn, 'Exclusive: Apple dropped plan for encrypting backups after FBI complained - sources' (2021) Reuters <https://www.reuters.com/article/us-apple-fbi-icloud-exclusive-idUSKBN1ZK1CT> accessed August 10th 2021.
} 
individuals depicted in them, the hashes of those images would therefore also potentially be the personal data of those individuals ${ }^{55}$. And, as with the images that the hashes represent, the hashes themselves would also be personal data relating to the iCloud account holder.

3. Safety vouchers. Safety vouchers contain a payload (an encrypted representation of the scanned image and the NeuralHash) and header (derived from that image's NeuralHash) and they cryptographically encode the result of the hash matching process. As with the image and the NeuralHash, the safety voucher (including its header and payload) is associated with a particular iCloud account and would therefore be personal data relating to the account holder. Things are more complicated for any people depicted in underlying image. In my view, whether the safety voucher is personal data relating to those people will depend on whether the contents of the payload - the NeuralHash and the representation of the image - is personal data relating to them. If a hash match is negative, the payload would be encrypted in such a way that - absent infinite time and computing power - no key will decrypt it (according to Apple, even Apple is not capable of decrypting the payload in those circumstances ${ }^{56}$ ). It is therefore not reasonably likely that people could be identified from the representation of the underlying image contained in that payload. If the hash match is positive, however, then the payload may be decrypted and inspected by Apple if at some point the iCloud user who holds the image crosses the threshold number of positive matches. It is therefore reasonably likely that individuals depicted in the representation of the underlying image contained in positive match payloads could be identified or identifiable at some point in future. The upshot of this is that payloads (and thus safety vouchers) for positive hash matches may be personal data relating to any people depicted in the scanned image, while safety vouchers for negative matches are not.

Images scanned by the CSAM Detection system prior to upload to iCloud, hashes of those images, and safety vouchers would thus all be information that relates to iCloud users. They would be associated with iCloud users' accounts, from which those users could be identified. They would therefore be personal data relating to those iCloud account holders. The scanned images (and potentially their hashes) would also be personal data relating to any living people depicted in them. It is also possible that safety vouchers would be personal data relating to any living people depicted in the representation of the underlying image contained within them, depending on whether the image's hash match was positive or negative.

\footnotetext{
${ }^{55}$ For a fuller exploration of whether hashes can be personal data, see Michèle Finck and Frank Pallas, 'They Who Must Not Be Identified - Distinguishing Personal from Non-Personal Data Under the GDPR' (2020) 10 International Data Privacy Law <https://doi.org/10.1093/idpl/ipz026> accessed August 10th 2021.

${ }^{56}$ Apple, Technical Summary (n 2), p.10-11.
} 
Finally, I'll note here that there are some kinds of processing that GDPR explicitly does not cover $^{57}$. These include things such as processing by individuals for purposes that are purely personal or household-related; they include activities which fall outside of the scope of EU law; and they include processing by law enforcement agencies ("competent authorities") for the purposes of preventing, investigating, detecting, or prosecuting criminal offences (governed instead by the Law Enforcement Directive ${ }^{58}$ ). Apple's CSAM Detection system would not fall within any of these excluded kinds of processing - Apple is not a law enforcement agency, for example.

Apple's CSAM Detection system would therefore process personal data within the material scope of GDPR.

\subsection{Territorial scope}

GDPR applies to:

(1) processing by a controller established in the EU, whether or not that processing takes place in the $\mathrm{EU}^{59}$, and

(2) processing by a controller not established in the EU of personal data relating to people located in the EU, where that processing is related to offering goods and services to people located in the EU or to monitoring the behaviour of people located in the $\mathrm{EU}^{60}$.

For now, Apple has only said that it is rolling out CSAM Detection in the United States. However, as I indicate above, my analysis precedes on the basis of a hypothesised future roll-out in the EU. For my purposes, then, I will assume that the processing I'm discussing is taking place for iCloud users located in the EU. Apple has a European establishment - Apple Distribution International Limited. This is incorporated in Ireland (which just happens to have a friendly tax regime). Even if Apple did not have an EU establishment, CSAM Detection deployed in the EU would constitute monitoring the behaviour of people located in the EU. CSAM detection rolled out in Europe would therefore fall within GDPR's territorial scope.

\footnotetext{
${ }^{57}$ GDPR art 2(2).

${ }^{58}$ Directive (EU) 2016/680 of the European Parliament and of the Council of 27 April 2016 on the protection of natural persons with regard to the processing of personal data by competent authorities for the purposes of the prevention, investigation, detection or prosecution of criminal offences or the execution of criminal penalties, and on the free movement of such data, and repealing Council Framework Decision 2008/977/JHA (2016) OJ L 119/89.

${ }^{59}$ GDPR art 3(1).

${ }^{60}$ GDPR art 3(2).
} 


\section{Who would be the data controller?}

The actor primarily responsible for ensuring that personal data processing complies with the law is known as the 'data controller'. Data controllers have a range of obligations around data processing - such as using appropriate technical and organisational measures to ensure that processing complies with GDPR and that they can demonstrate their compliance ${ }^{61}$; ensuring that personal data is only processed for particular purposes ${ }^{62}$ and deleted once it is no longer needed for those purposes ${ }^{63}$; using technical and organisational security measures appropriate to the risks to rights and freedoms posed by their processing ${ }^{64}$; ensuring data protection by design and by default; and more.

According to GDPR, the data controller is: "the natural or legal person, public authority, agency or other body which, alone or jointly with others, determines the purposes and means of the processing of personal data" ${ }^{\prime 25}$. What does this mean? Put simply, the data controller is the entity that determines why personal data is processed (the 'purposes') and how that data will be processed (the 'means'). It should be noted that determining who is the data controller for processing depends on the factual situation - i.e. who is actually determining why and how data is being processed - rather than something that can be definitively assigned in contract, terms of service, or some other arrangement (though these can inform an analysis of controllership).

In some cases, more than one entity will play a role in determining these things. There will then be multiple data controllers. They can either act together as joint controllers (where they jointly determine the purposes and means) or as multiple separate controllers (where, for instance, they each have their own purpose for the same processing).

An important point here is that it's not necessary that the controller actually processes the data on their own equipment. They can, for instance, delegate this processing to another entity. In that case, that other entity will be a 'data processor' ${ }^{66}$. Data processors process personal data on behalf and under the instruction of data controllers ${ }^{67}$.

In the case of on-device CSAM scanning, however, Apple wouldn't be delegating the processing to another entity in the sense of using a data processor. Apple would instead be running software on end-user's devices to do the processing. That is to say, though the

\footnotetext{
${ }^{61}$ GDPR art 24.

62 GDPR art 5(1)(b).

${ }^{63}$ GDPR art 5(1)(e).

${ }^{64}$ GDPR art 32.

${ }^{65}$ GDPR art 4(7).

${ }^{66}$ GDPR art 4(8).

${ }^{67}$ GDPR arts 4(8) and 28.
} 
actual computation would be undertaken on user's devices, it would ultimately be Apple's software that's doing it.

Given this, would Apple determine the purposes and means of processing? I'll take each of these in turn.

\subsection{The purposes of processing}

Ultimately, Apple has decided that it wants to scan iCloud users' images for CSAM so as to assist child safety organisations. It has developed a system that processes personal data for that stated purpose. As Apple says in its Technical Summary, "CSAM Detection enables Apple to accurately identify and report iCloud users who store known Child Sexual Abuse Material (CSAM) in their iCloud Photos accounts" ${ }^{68}$. So this is straightforward: Apple would determine the purposes of the processing involved in its CSAM Detection system.

\subsection{The means of processing}

Some regulatory bodies (such as the European Data Protection Board ${ }^{69}$ ) have suggested that the law might distinguish between the 'essential' means (such as questions like 'whose data will be processed?, 'which data will be processed?', 'for how long?', 'who will have access to the data?') and the 'non-essential' means (relating to more practical, technical questions such as which software or hardware will be used). According to the European Data Protection Board (EDPB), processors can determine the non-essential means without compromising their status as a processor, while only controllers can determine the essential means $^{70}$.

I should say that it's not exactly clear how these regulatory bodies have arrived at the concepts of 'essential' and 'non-essential' means as a way of distinguishing between controllers and processors. Nevertheless, whether or not they in fact offer a way of distinguishing in law between controllers and processors, these concepts do provide a useful way to think about the different things that Apple is doing in its CSAM Detection system.

In terms of the 'essential' means, Apple would determine whose data would be processed (iCloud users). Apple would determine which data would be processed (images to be uploaded to iCloud, hashes related to those images, safety vouchers). Apple would determine for how long this data would be processed (the safety voucher would, as far as I

\footnotetext{
${ }^{68}$ Apple, Technical Summary (n 2), p.3.

${ }^{69} \mathrm{~A}$ body established under GDPR to coordinate the activities of data protection regulators in the Member States and to advise on the interpretation of data protection law (GDPR art 68).

${ }^{70}$ European Data Protection Board, 'Guidelines 07/2020 on the concepts of controller and processor in the GDPR' (2020), pp.13-15.
} 
can tell, be retained for as long as Apple deems necessary). Apple would determine who has access to the data (Apple and their employees and, when Apple determines a user has crossed the threshold set by Apple, the NCMEC, or whichever EU-based equivalent Apple would choose if it deployed CSAM Detection in the EU).

In terms of the 'non-essential' means, Apple's CSAM Detection system involves software (NeuralHash, Private Set Intersection, and Threshold Secret Sharing) developed internally by Apple, running on hardware developed by Apple (iCloud users' iPhones) and on cloud servers owned or managed by Apple. Apple's documentation (such as their Technical Summary) is clear that Apple has chosen these technologies as the mechanism by which it believes it can do on-device CSAM scanning while respecting user privacy ${ }^{71}$. The flaws with the claim that this preserves user privacy aside, Apple therefore itself views this as a system that it has developed that allows it to scan for CSAM in its preferred way.

Whether or not we follow the EDPB's guidance and distinguish between 'essential' and 'non-essential' means, we can conclude from the above that Apple would indeed determine the means of the processing involved in both the on-device and on-server aspects of its CSAM Detection system.

\subsection{Would Apple be the controller for its CSAM Detection system?}

Following the above analysis, my view is that Apple would determine both the purposes and the means for the personal data processing involved in its CSAM Detection system. That the NeuralHash and some of the Private Set Intersection aspects of the CSAM Detection system operate on end-user's devices does not affect Apple's position in law - wherever the processing is actually happening, Apple would determine its purposes and means. Apple would therefore be the controller for that processing, both on-device and on-server.

\section{What legal bases might be available?}

Under GDPR, data controllers must have a valid basis in law for all processing of personal data $^{72}$. I will briefly consider here whether any of those might apply to Apple's CSAM Detection processing. Because Apple's CSAM Detection system would involve on-device processing of images stored on iCloud users' iPhones, I will also consider the effect of relevant provisions of the ePrivacy Directive.

\footnotetext{
${ }^{71}$ Apple, Technical Summary (n 2).

72 GDPR arts 6 and 9.
} 


\subsection{Legal bases for processing under GDPR}

As indicated above, to be lawful, personal data processing must meet one of the conditions specified in GDPR. Though the consent of the data subject is probably the most well-known of these, it is by no means the only potential legal basis.

In fact, Article 6 GDPR sets out six possible bases ${ }^{73}$ - as well as where the data subject has consented, processing may be lawful where it is necessary for the performance of a contract to which the data subject is a party; where it is necessary to comply with a legal obligation to which the controller is subject; where it is necessary to protect the vital interests of the data subject or another person; where it is necessary for the performance of a task carried out in the public interest or in the exercise of official authority vested in the controller; or where it is necessary for the legitimate interests of the controller.

In principle, several of these seem like they might be relevant to CSAM Detection. Arguably, CSAM Detection could help protect the vital interests of the children depicted in those images (though GDPR does say that the 'necessary to protect the vital interests of another person' basis should only be used when other bases aren't available ${ }^{74}$ ). Similarly, Apple might argue that CSAM Detection is carried out in the public interest (however, this requires a basis in EU or Member State law ${ }^{75}$ - that is to say, a law authorising or mandating that processing). Apple may even argue that detecting CSAM uploaded to its servers is in its legitimate interests (though the controller's legitimate interests may be overridden by the interests or fundamental rights and freedoms of data subjects, including those data subjects' privacy and data protection rights ${ }^{76}$ ).

I should note, though, that 'necessary' is a high bar - it doesn't mean 'helpful', 'beneficial', or 'makes it easier'. Processing can only be 'necessary' when objective evidence shows that there is no alternative means of achieving the same result ${ }^{77}$. The various grounds that require a necessity test (that is, all except consent) may therefore not be applicable here - it is not clear that CSAM Detection would pass that test. Apple might argue that on-device hash matching is necessary to determine whether CSAM is being held by users. But other cloud storage providers - such as Microsoft - perform on-server analysis for the same purpose, so processing on-device is clearly not necessary. Moreover, as indicated in §3.1, Apple's iCloud Photos service is not end-to-end encrypted, so Apple could feasibly do the whole CSAM detection process on-server.

\footnotetext{
73 GDPR art 6(1).

74 GDPR recital 46.

75 GDPR recital 45.

${ }^{76}$ GDPR art 6(1)(f).

${ }^{77}$ European Data Protection Supervisor, 'Assessing the necessity of measures that limit the fundamental right to the protection of personal data: A Toolkit' (2017) < https://edps.europa.eu/sites/edp/files/publication/1704-11 necessity toolkit en 0.pdf> accessed 8th August 2021.
} 
Perhaps the best bet for Apple might be the 'legitimate interests' basis. Apple might argue that the on-device aspects of CSAM Detection are necessary to prevent CSAM being uploaded to its iCloud servers. That said, given that user images that match with known CSAM will seemingly be uploaded to iCloud anyway ${ }^{78}$, and given that Apple's iCloud-related hosting would ordinarily be shielded from liability for CSAM by the eCommerce Directive ${ }^{79}$ (provided Apple had no knowledge or awareness of illegal material ${ }^{80}$ ), it's not clear which of Apple's legitimate interests the processing would actually be necessary for ${ }^{81}$. Nor is it clear that Apple's interest would not be overridden by data subjects' fundamental rights, in particular to privacy ${ }^{82}$ and data protection ${ }^{83}$.

\subsection{The ePrivacy Directive}

In any case, because Apple's CSAM Detection system access images on-device, we also have to consider the interaction of GDPR with the ePrivacy Directive. The ePrivacy Directive is, as the name indicates, a Directive - a type of EU law that needs to be implemented by each Member State for it to apply in their domestic law (this contrasts with a Regulation - such as GDPR - which takes effect in each Member State automatically). There may therefore be variations depending on the specifics of national implementations, but I will use the text of the ePrivacy Directive itself as the primary source for determining what EU ePrivacy law says.

\footnotetext{
${ }^{78}$ Apple's Technical Summary implies that all images are uploaded to iCloud, along with the safety voucher containing the results of the hash matching (Apple, Technical Summary (n 2), p.3). The on-server part of the system only flags CSAM for manual review when a threshold is crossed.

${ }^{79}$ Directive 2000/31/EC of the European Parliament and of the Council of 8 June 2000 on certain legal aspects of information society services, in particular electronic commerce, in the Internal Market [2000] OJ L 178/1 ('ECommerce Directive') art 14.

${ }^{80} \mathrm{E}$-Commerce Directive art 14.

${ }^{81}$ The hash matching aspects of its CSAM Detection system may even risk opening Apple to liability for images that are hash matched with CSAM. The E-Commerce Directive's hosting liability shield is conditional on service providers - such as Apple - not having actual knowledge or awareness of illegal activity or information. If the service provider is "aware of facts or circumstances from which the illegal activity or information is apparent"(E-Commerce Directive art 14), then it must expeditiously remove or disable access to that information to maintain its liability protection. Such 'facts or circumstances' might well include a positive hash match with known CSAM. Yet Apple's Technical Summary says that, in its CSAM Detection system, "Users can't identify which images were flagged as CSAM by the system" (Apple, Technical Summary (n 2), p.3), and the system more generally only flags CSAM for review when a threshold is crossed. The implication of this is that CSAM images are not removed when flagged, nor is access disabled. They are instead uploaded to iCloud, along with the safety voucher. However, the results of hash matching are cryptographically encoded in the safety voucher before upload. Apple would presumably argue that Threshold Secret Sharing means that it cannot see the contents of safety vouchers stored on iCloud and is therefore not actually aware of CSAM until the threshold is crossed, therefore maintaining its liability protection. I don't consider this issue in any further depth, as it is incidental to the main GDPR analysis.

${ }^{82}$ Charter of Fundamental Rights of the European Union [2012] OJ C326/391 ('EU Charter') art 7.

${ }^{83}$ EU Charter art 8.
} 
The ePrivacy Directive is what is known as 'lex specialis'. That is to say, it is law applying to some specific activities and overriding general law. In this case, the ePrivacy Directive particularises more general data protection law (i.e. GDPR) and overrides GDPR where it applies.

Article 5(3) of the ePrivacy Directive says:
"Member States shall ensure that the storing of information, or the gaining of access to information already stored, in the terminal equipment of a subscriber or user is only allowed on condition that the subscriber or user concerned has given his or her consent, having been provided with clear and comprehensive information, in accordance with Directive 95/46/EC, inter alia, about the purposes of the processing." ${ }^{84}$

I'll unpack a few things in this. In plain language, 'user' for our purposes means a person using a service that offers internet access for private or business purposes ${ }^{85}$. 'Terminal equipment' means a device connected to a public network (such as the internet) to send, process, or receive information ${ }^{86}$. The "terminal equipment of a subscriber or user" would thus, in the context of Apple's CSAM Detection system, be an iPhone connected to the internet, either through a mobile or fixed connection. That is to say, iPhones are terminal equipment for the purposes of the ePrivacy Directive. Access to information on those iPhones thus requires the user's consent.

The importance of protecting information on users' terminal equipment is highlighted in the ePrivacy Directive's recitals:

"Terminal equipment of users of electronic communications networks and any information stored on such equipment are part of the private sphere of

\footnotetext{
84 ePrivacy Directive art 5(3).

${ }^{85}$ The actual definition of 'user' in the ePrivacy Directive is "any natural person using a publicly available electronic communications service, for private or business purposes, without necessarily having subscribed to this service" (ePrivacy Directive art 2(a)). 'Electronic communications service' is in turn defined in the European Electronic Communications Code to mean "a service normally provided for remuneration via electronic communications networks", encompassing 'internet access services', 'interpersonal communications services', and 'services consisting wholly or mainly in the conveyance of signals such as transmission services used for the provision of machine-to-machine services and for broadcasting' (European Electronic Communications Code art 2(4)). The Regulation on Open Internet Access defines 'internet access service' as "a publicly available electronic communications service that provides access to the internet, and thereby connectivity to virtually all end points of the internet, irrespective of the network technology and terminal equipment used" (Regulation (EU) $2015 / 2120$ art 2(2)).

${ }^{86}$ Commission Directive 2008/63/EC of 20 June 2008 on competition in the markets in telecommunications terminal equipment (Codified version) (Text with EEA relevance).
} 
the users requiring protection under the European Convention for the

Protection of Human Rights and Fundamental Freedoms." 87

The fact that Apple would be using on-device hash matching - necessarily accessing information stored on device - is crucial here. Essentially, the ePrivacy Directive says that if Apple wants to perform on-device CSAM hash matching on images stored on an iCloud user's iPhone then it must have that user's consent. Because the ePrivacy Directive is lex specialis, particularising the law in this area, Article 5(3) ePrivacy Directive overrides Article 6 GDPR with regard to the subject matter of Article 5(3). That is to say, the consent requirement in Article 5(3) ePrivacy Directive excludes any of the non-consent legal bases provided for in Article 6 GDPR (such as 'legitimate interests' or 'vital interests', discussed above). Consent would therefore be the only available legal basis for the on-device aspects of Apple's CSAM Detection system.

\subsection{What should consent involve?}

The Directive says that users should be provided with "clear and comprehensive information", in accordance with Directive 95/46/EC, including about the purposes of the processing (in this case, CSAM detection). 'Directive 95/46/EC' was 1995's Data Protection Directive $^{88}$, which was replaced by GDPR. References in the ePrivacy Directive to the Data Protection Directive are to be read as references to GDPR ${ }^{89}$.

Moreover, recital 17 of the ePrivacy Directive says:

"For the purposes of this Directive, consent of a user or subscriber, regardless of whether the latter is a natural or a legal person, should have the same meaning as the data subject's consent as defined and further specified in Directive 95/46/EC. Consent may be given by any appropriate method enabling a freely given specific and informed indication of the user's wishes, including by ticking a box when visiting an Internet website." 90

Recitals are not themselves binding, but they provide a guide to interpretation of the law's binding provisions.

\footnotetext{
87 ePrivacy Directive recital 24.

${ }^{88}$ Directive 95/46/EC of the European Parliament and of the Council of 24 October 1995 on the protection of individuals with regard to the processing of personal data and on the free movement of such data [1995] OJ L281/31 ('Data Protection Directive').

89 GDPR art 94.

90 ePrivacy Directive recital 17.
} 
The CJEU held in 2018 that requests for consent under the ePrivacy Directive - such as required by Article 5(3) - must meet GDPR's standards for consent ${ }^{91}$ (even if the information being accessed on user's terminal equipment isn't actually personal data ${ }^{92}$ ). GDPR says that consent must be "freely given, specific, informed, and unambiguous" 93 . Consent must be opt-in (a "clear affirmative action"94), rather than opt-out ${ }^{95}$. There must be a real option to refuse consent, and to withdraw consent once given ${ }^{96}$; consent is not 'freely given', according to GDPR's recitals, "if the data subject has no genuine or free choice or is unable to refuse or withdraw consent without detriment" ${ }^{\prime 97}$.

Importantly, because consent must be specific, consent to one purpose (such as processing to detect CSAM) can't be presumed from consent to another purpose (such as processing for cloud backup) ${ }^{98}$. Nor can consent to processing to detect CSAM be 'bundled' with consent to cloud backup. GDPR strongly indicates that 'bundling' - the practice of making access to a service conditional on giving consent to processing that is not necessary for that service - is not permitted: Article 7 GDPR says:

"When assessing whether consent is freely given, utmost account shall be taken of whether, inter alia, the performance of a contract, including the provision of a service, is conditional on consent to the processing of personal data that is not necessary for the performance of that contract" 99

Recital 43 GDPR says:

"Consent is presumed not to be freely given if [...] the performance of a contract, including the provision of a service, is dependent on the consent despite such consent not being necessary for such performance." 100

Rather, users should be able to refuse or withdraw consent "without detriment"101. Again, recitals are not binding, but they do guide interpretation of the law's binding provisions. Here, recital 43 tells us how Article 7 should be interpreted: we should presume that

\footnotetext{
${ }^{91}$ Case C-673/17 Bundesverband der Verbraucherzentralen und Verbraucherverbände - Verbraucherzentrale Bundesverband e.V. v Planet49 GmbH [2018] ('Planet49').

92 Planet49 paras 66-71.

93 GDPR art 4(11), recital 32.

${ }^{94}$ GDPR art 4(11), recital 32.

95 Planet49.

${ }^{96}$ GDPR art 7(3).

97 GDPR recital 42.

98 Planet49 para 58; GDPR recital 32.

${ }^{99}$ GDPR art 7(4).

100 GDPR recital 43.

${ }^{101}$ GDPR recital 32.
} 
consent was not freely given (and is therefore not valid) if provision of a service is made conditional on consent to processing that is not necessary for that service.

\subsection{What does all this mean?}

All this is to say that, due to the interaction between the ePrivacy Directive, Apple would need the consent of iPhone users for the on-device aspects of its CSAM Detection system. Such consent must be opt-in, rather than out-out. Apple must provide a real option to refuse consent, without making access to iCloud dependent on it, and must allow iPhone users who have consented to later withdraw that consent. In practice, this should mean that iPhone users can refuse to permit Apple to perform on-device CSAM Detection without losing access to iCloud.

\subsection{What about the rest of the CSAM Detection system?}

The discussion so far in $\S 5$ covers on-device processing of data stored on iCloud users' iPhones. However, as indicated in $§ 2$, Apple’s CSAM Detection system consists of three components: first, on-device hash matching; second, on-server aspects of hash matching and determination of whether the threshold has been crossed; and third, manual review of safety vouchers. Access to data stored on iPhones is part of on-device hash-matching - a necessary first step that enables the rest of the CSAM Detection system.

Remember that lex specialis only overrides general law in relation to its specific subject matter. Because Article 5(3) ePrivacy Directive only covers the on-device component of Apple's CSAM Detection system (in that it relates solely to access to information stored on users' terminal equipment ${ }^{102}$ ), it would therefore only override Article 6 GDPR in relation to that on-device processing. Apple would thus need to also have a legal basis in Article 6 GDPR itself for the subsequent on-server and manual review components of CSAM Detection.

However, the interaction between GDPR and the ePrivacy Directive is still relevant here. As I discuss above (§5.3), consent under the ePrivacy Directive has the same meaning and requirements as consent under $\mathrm{GDPR}^{103}$. In relation to the on-device component of CSAM Detection, Apple would therefore need to obtain consent for specific, clearly and explicitly stated purposes, having comprehensively explained to users what it intends to do with their data and for what purposes it may be processed ${ }^{104}$. Importantly, GDPR (in its recitals) says that "Consent should cover all processing activities carried out for the same purpose or

\footnotetext{
102 ePrivacy Directive art 5(3).

103 ePrivacy Directive art 5(3), recital 17; Planet49.

${ }^{104}$ GDPR arts 4(11) and 7, recitals 32 and 42-43; ePrivacy Directive art 5(3), recital 17.
} 
purposes"105. This, I would argue, means that any consent obtained by Apple to on-device processing for the purpose of CSAM Detection should also explicitly cover all other processing activities associated with CSAM Detection, including those that take place offdevice (i.e. on-server and manual review).

Since Apple would need to ask for consent to off-device processing when it asks for consent to on-device processing for the purpose of CSAM Detection, Apple should not then rely on a different (i.e. non-consent) legal basis under Article 6 GDPR for any off-device processing for that same purpose ${ }^{106}$. By requiring consent to access information stored on users' iPhones for the purpose of CSAM Detection, the ePrivacy Directive would therefore have the practical effect of restricting the available Article 6 GDPR legal bases for the processing that constitutes the other (off-device) components of the CSAM Detection system. The appropriate legal basis under Article 6 GDPR for the processing activities in those latter offdevice components of the CSAM Detection system would thus be the initial consent obtained by Apple for CSAM Detection.

\subsection{Some further complications}

As I discussed above (§3.1), the images (and potentially the hashes and safety vouchers) processed in Apple's CSAM detection system would not only be the iCloud user's personal data, but also the personal data of any individuals depicted in them. This complicates things further for Apple in ways that are not clear. Consent under GDPR is obtained from individual data subjects - that is to say, where a data controller is relying exclusively on data subjects' consent, they would need to obtain the consent of each data subject. Though Apple could request the consent of iPhone users, it would likely not be able to request the consent of other people depicted in scanned images.

Notably, though, the ePrivacy Directive only says that consent of the iPhone user would be required ${ }^{107}$. In relation to other data subjects not covered by the ePrivacy Directive, the Directive would perhaps not override Article 6 of GDPR. Apple may therefore be able to rely on GDPR's other legal bases for processing in relation to other data subjects (such as where the processing is necessary for Apple's legitimate interests). However, I admit to being

\footnotetext{
105 GDPR recital 32.

106 See European Data Protection Board, 'Guidelines 05/2020 on consent under Regulation 2016/679' (2020), paras $121-123$

<https://edpb.europa.eu/sites/default/files/files/file1/edpb guidelines 202005 consent en.pdf> accessed August 9th 2021; European Data Protection Board, 'Opinion 5/2019 on the interplay between the ePrivacy Directive and the GDPR, in particular regarding the competence, tasks and powers of data protection authorities' (2019), pp.13-14 $<$ https://edpb.europa.eu/sites/default/files/files/file1/201905 edpb opinion eprivacydir gdpr interplay en 0.pdf> accessed August 9th 2021.

107 ePrivacy Directive art 5(3).
} 
uncertain exactly how GDPR and the ePrivacy Directive interact in relation to data subjects who are not the iPhone user in this particular context.

I do not explore this question further here, as, regardless of what legal basis Apple relies on for other data subjects, Apple would need to obtain the consent of the iPhone user to ondevice CSAM Detection, as described above - and the iPhone user would be the target of the CSAM Detection system.

\section{On-device CSAM detection - the next big legislative battle?}

Based on the above, EU data protection and ePrivacy law provides some pretty significant stumbling blocks for Apple's CSAM Detection system were they to decide to deploy it in the EU. It is unlikely in the extreme that even minimally informed holders of CSAM would give consent to Apple's CSAM Detection system. The principal argument in favour of CSAM Detection - that it would help tackle CSAM - would therefore largely fall away. We would instead be left with a situation where the only people subjecting themselves to this surveillance could conceivably be people who may never encounter CSAM at all. Yet, in rolling out CSAM Detection, Apple would have taken on the responsibilities, obligations, and legal risks of a data controller for its CSAM Detection processing. I suggest, therefore, that it is quite possible - perhaps even likely - that Apple will refrain from deploying this system in the EU while the law remains as it is.

(Incidentally, Article 5(3) of the ePrivacy Directive is the provision that led to the widespread and widely bemoaned cookie consent requests. Perhaps some of its critics will now recognise the importance of a law that prevents others from storing or accessing information on your device without your consent).

Those of us who are concerned about the impact of Apple's CSAM Detection system on fundamental rights to privacy and data protection, as well as about the precedent set by a 'Big Tech' company voluntarily implementing such a system, should not rest easy, however. The law may - for now - prevent Apple, Google, Microsoft, Amazon, Facebook, or anyone else from doing this kind of on-device CSAM scanning in any way that is likely to actually identify those holding CSAM. But laws are not set in stone. Policymakers may be swayed Apple's claims about protecting privacy and their fancy cryptography. The EU may yet remove the requirement to obtain users' consent to access on-device information and can do so with ordinary legislation. Moreover, Member States can themselves set aside that requirement of consent for access to on-device information where doing so is necessary, 
appropriate, and proportionate in a democratic society for the purposes of prevention, investigation, detection, and prosecution of criminal offences ${ }^{108}$.

The prospect of either the EU or its Member States changing the law to permit companies like Apple to do on-device CSAM detection without users' consent is not far-fetched. As I indicated in the introduction ( $§ 1)$, the EU has already passed legislation ${ }^{109}$ allowing numberindependent messaging services (such as Facebook Messenger), to bypass the ePrivacy Directive's requirement ${ }^{110}$ to obtain users' consent to scan their messages for CSAM. Towards the end of 2021, the EU will announce legislation that may make CSAM scanning mandatory for some messaging services ${ }^{111}$. This followed a public consultation that closed earlier in 2021. Whether this will require on-device scanning - in particular, for end-to-end encrypted message services - remains unclear.

The EU is also in the process of replacing the ePrivacy Directive with an ePrivacy Regulation, which will take effect automatically in the Member States and harmonise the law in this area. Though the draft Regulation has been working its way through the legislative process since it was first introduced in 2017, it entered 'trilogue' in May 2021 (trilogue is the process by which the European Commission, European Parliament, and EU Council attempt to hash out the differences in their preferred versions of proposed legislation to come to an agreement on a final text). The current Council draft of the proposed ePrivacy Regulation would not remove the consent requirement for on-device CSAM detection ${ }^{112}$, but it is still a draft and subject to change.

If, like me, you think that Apple's CSAM Detection system is bad news, then such moves should be strongly resisted. Since we already live in a world of intrusive mass surveillance by companies and by governments - some might think it anachronistic, obstinate, or even foolish to insist on privacy and data protection in the face of a crime as heinous as child sexual abuse. But interferences with such fundamental rights - even for something like CSAM - must be limited to what is strictly necessary and proportionate in a democratic society. Democracy and free society are built on our shared fundamental rights and values. We cannot abandon these most important things to embrace the solutionist logics of

\footnotetext{
108 ePrivacy Directive art 15(1).

${ }^{109}$ Regulation (E) 2021/1232 of the European Parliament and of the Council of 14 July 2021 on a temporary derogation from certain provisions of Directive 2002/58/EC as regards the use of technologies by providers of number-independent interpersonal communications services for the processing of personal and other data for the purpose of combating online child sexual abuse [2020] OJ L 274/41.

110 ePrivacy Directive art 5(1).

${ }^{111}$ European Commission, 'Fighting child sexual abuse: detection, removal and reporting of illegal content online' <https://ec.europa.eu/info/law/better-regulation/have-your-say/initiatives/12726-Fighting-childsexual-abuse-detection-removal-and-reporting-of-illegal-content-online en> accessed August 9th 2021.

112 Proposal for a Regulation of the European Parliament and of the Council concerning the respect for private life and the protection of personal data in electronic communications and repealing Directive 2002/58/EC (Regulation on Privacy and Electronic Communications) art 8 (draft as of January 2021: https://data.consilium.europa.eu/doc/document/ST-5008-2021-INIT/en/pdf).
} 
technologies where the efficacy is so unproven, the potential benefit so uncertain, the potential for abuse so real, and the risks to rights and freedoms so clear.

None of this is to downplay the importance of tackling CSAM. None of this is to downplay the seriousness of its harm. Nobody concerned about what Apple is proposing to do lacks understanding of the arguments in its favour or empathy with victims and survivors. It is instead to plead that we do not rush headlong into illusory quick fixes that crack open a door that may never again be shut. Few would argue that more is not needed to tackle CSAM. But something must be done cannot mean anything must be done. It should not mean that this must be done. 